\title{
Weak monotonicity and Bayes-Nash incentive compatibility
}

Citation for published version (APA):

Müller, R. J., Perea ý Monsuwé, A., \& Wolf, S. (2005). Weak monotonicity and Bayes-Nash incentive compatibility. METEOR, Maastricht University School of Business and Economics. METEOR Research Memorandum No. 040 https://doi.org/10.26481/umamet.2005040

Document status and date:

Published: 01/01/2005

DOI:

10.26481/umamet.2005040

Document Version:

Publisher's PDF, also known as Version of record

\section{Please check the document version of this publication:}

- A submitted manuscript is the version of the article upon submission and before peer-review. There can be important differences between the submitted version and the official published version of record.

People interested in the research are advised to contact the author for the final version of the publication, or visit the DOI to the publisher's website.

- The final author version and the galley proof are versions of the publication after peer review.

- The final published version features the final layout of the paper including the volume, issue and page numbers.

Link to publication

\footnotetext{
General rights rights.

- You may freely distribute the URL identifying the publication in the public portal. please follow below link for the End User Agreement:

www.umlib.nl/taverne-license

Take down policy

If you believe that this document breaches copyright please contact us at:

repository@maastrichtuniversity.nl

providing details and we will investigate your claim.
}

Copyright and moral rights for the publications made accessible in the public portal are retained by the authors and/or other copyright owners and it is a condition of accessing publications that users recognise and abide by the legal requirements associated with these

- Users may download and print one copy of any publication from the public portal for the purpose of private study or research.

- You may not further distribute the material or use it for any profit-making activity or commercial gain

If the publication is distributed under the terms of Article $25 \mathrm{fa}$ of the Dutch Copyright Act, indicated by the "Taverne" license above, 


\title{
Weak Monotonicity and Bayes-Nash Incentive Compatibility
}

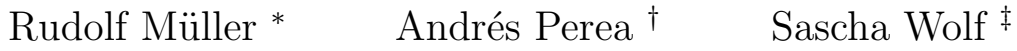

September 7, 2005

\begin{abstract}
An allocation rule is called Bayes-Nash incentive compatible, if there exists a payment rule, such that truthful reports of agents' types form a Bayes-Nash equilibrium in the direct revelation mechanism consisting of the allocation rule and the payment rule. This paper provides characterizations of Bayes-Nash incentive compatible allocation rules in social choice settings where agents have one-dimensional or multi-dimensional types, quasi-linear utility functions and interdependent valuations. The characterizations are derived by constructing complete directed graphs on agents' type spaces with cost of manipulation as lengths of edges. Weak monotonicity of the allocation rule corresponds to the condition that all 2-cycles in these graphs have non-negative length.

For one-dimensional types and agents' valuation functions satisfying non-decreasing expected differences, we show that weak monotonicity of the allocation rule is a necessary and sufficient condition for the rule to be Bayes-Nash incentive compatibile. In the case where types are multi-dimensional and the valuation for each outcome is a linear function in the agent's type, we show that weak monotonicity of the allocation rule together with an integrability condition is a necessary and sufficient condition for Bayes-Nash incentive compatibility.
\end{abstract}

Keywords: Mechanism Design, Incentive Compatibility, Weak Monotonicity

JEL: C72

${ }^{*}$ Department of Quantitative Economics, Maastricht University, P.O. Box 616, 6200 MD Maastricht, The Netherlands; e-mail: r.muller@ke.unimaas.nl

${ }^{\dagger}$ Corresponding author. Department of Quantitative Economics, Maastricht University, P.O. Box 616, 6200 MD Maastricht, The Netherlands; e-mail: a.perea@ke.unimaas.nl

${ }^{\ddagger}$ Department of Quantitative Economics, Maastricht University, P.O. Box 616, 6200 MD Maastricht, The Netherlands; e-mail: s.wolf@ke.unimaas.nl 


\section{Introduction}

This paper is concerned with the characterization of Bayes-Nash incentive compatible allocation rules in social choice settings where agents have independently distributed, onedimensional or multi-dimensional types and quasi-linear utility functions, that is, utility is the valuation of an allocation minus a payment. We allow for interdependent valuations across agents. The central task addressed in this paper is the following: given such type distributions and valuations, characterize precisely those allocation rules for which there exists a payment rule such that truthful reporting of agent's types forms a Bayes-Nash equilibrium in the direct revelation mechanism consisting of the allocation rule combined with the payment rule. In addition, we aim for a framework that lets us construct a payment rule, if any, which makes a particular allocation rule Bayes-Nash incentive compatible. For example, given an allocation rule which decides in a combinatorial auction for each set of bids for each agent which set of items he wins, we want to be able to decide whether there exists a pricing scheme for winning bids that makes truthful bidding a Bayes-Nash equilibrium. If the answer is yes, we would like to have means to construct such a pricing scheme.

\section{$1.1 \quad$ Related Work}

A recent stream of literature offers examples of characterizing dominant strategy incentive compatible allocation rules in terms of a monotonicity condition on the allocation rule, see e.g. Bikhchandani, Chatterji \& Sen [2] and Lavi, Mu'alem \& Nisan [8]. Gui, Müller \& Vohra [3] extend these results to larger classes of preference domains by making a link to network theory. The most general results are by Saks \& Yu [15], who show that previous results extend to any convex multi-dimensional type space. An allocation rule is dominant strategy incentive compatible, if there exists a payment rule such that for any report of the other agents an agent maximizes his own utility by reporting truthfully his type.

The environment considered by Saks \& Yu [15] features quasi-linear utilities and multidimensional types. The allocation rule maps agents' type reports into a finite set of $m$ possible outcomes. An agent's type is a vector in $\mathbb{R}^{m}$ reflecting his valuation of the different possible outcomes, that is, the agent's valuation of some outcome $a$ is given by the $a^{\text {th }}$ element of his type vector. Agents' type spaces are assumed to be convex. Saks \& Yu [15] show that dominant strategy incentive compatible allocation rules in this setting can be characterized 
in terms of weak monotonicity, a term introduced by Lavi, Mu'alem \& Nisan [8]. In order to derive this result they construct complete directed graphs in the following way: Take some agent and fix a profile of type reports for the others. Now, a directed graph is constructed by associating a node with each outcome and putting a directed edge between each ordered pair of nodes. Take two outcomes $a$ and $b$. Consider the difference of the valuation of $a$ and the valuation of $b$ with respect to every type for which truthfully reporting this type yields outcome $a$. The length of the network edge from $a$ to $b$ is defined as the infimum of all these differences. In this fashion a graph is constructed for every agent and every possible report profile of the other agents. Weak monotonicity states that for any two different outcomes $a$ and $b$, the sum of the two edge lengths from $a$ to $b$ and from $b$ to $a$ is non-negative.

Earlier, Rochet [12] characterized dominant strategy implementation in cases where the set of outcomes is not necessarily finite; an assumption that is crucial to the work of Saks \& $\mathrm{Yu}[15]$. For the case where agents have one-dimensional, convex type spaces and their valuation functions satisfy the increasing differences property, Rochet [12] shows that dominant strategy incentive compatibility can be characterized in terms of a monotonicity condition on the allocation rule alone. Next, he considers a setting where agents have multi-dimensional, convex type spaces and valuation functions which are linear w.r.t. their own true types. Taking some additional differentiability assumptions, Rochet [12] shows that in this case dominant strategy incentive compatibility can be characterized in terms of a monotonicity condition on the allocation rule plus an integrability condition.

Monotonicity has also been used to characterize Bayes-Nash incentive compatible allocation rules. Malakhov \& Vohra [10] consider an auction setting where multiple units of an indivisible good are auctioned off to several agents with constant marginal valuations. The one-dimensional type of an agent is his marginal valuation. Each agent has a finite set of possible types. Agents do not have interdependent valuations. Malakhov \& Vohra [10] show that Bayes-Nash incentive compatibility holds if and only if the allocation rule is monotone. Myerson [11] gives such a characterization for a single-item auction setting where agents have one-dimensional, convex type spaces and interdependent valuations.

Jehiel, Moldovanu \& Stacchetti [5] and Jehiel \& Moldovanu [4] develop characterizations for social choice settings where agents have multi-dimensional, convex type spaces and valuation functions which are linear w.r.t. their true types. Their characterizations of Bayes-Nash 
incentive compatibility include a monotonicity condition on the allocation rule as well as an integrability condition comparable to the one presented by Rochet [12].

\subsection{Our Contribution}

Similar to the network approach of Gui, Müller \& Vohra [3] and Saks \& Yu [15] we construct graphs. If an allocation rule is Bayes-Nash incentive compatible, then there exists a payment rule such that an agent's expected utility for truthfully reporting his type $t$ is at least as high as his expected utility for misreporting some type $s$. Similarly, an agent's expected utility for truthfully reporting type $s$ is at least as high as his expected utility for misreporting type $t$. From combining these two conditions we get a weak monotonicity condition on the allocation rule. This condition is the expected utility equivalent of the monotonicity condition mentioned in the context of dominant strategy incentive compatible allocation rules. Weak monotonicity is a necessary condition for Bayes-Nash incentive compatibility. It expresses that the expected gain in valuation for truthfully reporting $t$ instead of misreporting $s$ should be at least as big as the expected gain in valuation for misreporting $t$ instead of truthfully reporting $s$.

Recognizing that the constraints inherent in the definition of Bayes-Nash incentive compatibility have a natural network interpretation we build complete directed graphs for agents' type spaces. To do so we associate a node with each type and put a directed edge between each ordered pair of nodes. The length of the edge going from the node associated with type $s$ to the node associated with the type $t$ is defined as the cost of manipulation, that is, the expected difference in an agent's valuation for truthfully reporting $t$ instead of misreporting s. Note that unlike the network approach of Gui, Müller \& Vohra [3] and Saks \& Yu [15] (see description above) we construct only one graph for each agent since we work in terms of expectations and do not consider each possible type profile of the other agents separately. Furthermore, each of these graphs contains (except for the case of finite type spaces) an infinite number of nodes as we associate a node with each possible type of the agent. One could also construct outcome based graphs (as done by Gui, Müller \& Vohra [3] and Saks \& Yu [15]) by associating a node with each possible probability distribution over outcomes. However, these graphs possibly also contain an infinite number of nodes since we allow that the different possible type reports of an agent induce an infinite number of probability distributions over 
outcomes. For example, in the single-item auction framework presented in Section 4 it is quite natural to allow that every different type report of an agent yields a different expected conditional probability for him to win the object.

The outline of the paper is as follows: In Section 2 we state some basic assumptions and definitions. Throughout the paper we assume that agents have quasi-linear utility functions and independently distributed, privately known types. Furthermore, we allow for interdependent valuations. We do not put any restrictions on the number of possible outcomes.

In Section 3 we show that an allocation rule is Bayes-Nash incentive compatible if and only if the graphs described above contain no finite, negative length cycles. Rochet [12] shows that dominant strategy incentive compatibility can be characterized in terms of the absence of finite, negative length cycles in similar graphs. Our result is the Bayes-Nash equivalent for his finding. Furthermore, we show as a lemma that the costs of manipulation are decomposition monotone if the allocation rule satisfies weak monotonicity and agents' valuation functions satisfy non-decreasing expected differences (definitions can be found in Section 2). Using this result we can fold the aforementioned auction settings of Myerson [11] and Malakhov \& Vohra [10] into the framework presented in Section 4.

In Section 4 agents' types are restricted to be one-dimensional. No further restrictions on agents' type spaces are made, that is, we allow for infinite type spaces as considered by Myerson [11] as well as for finite type spaces as considered by Malakhov \& Vohra [10]. Without any additional restrictions this setting is too general in order for weak monotonicity to be a sufficient condition for Bayes-Nash incentive compatibility. This is illustrated by an example. However, for the case that the costs of manipulation are assumed to be decomposition monotone we are able to show that weak monotonicity is a necessary and sufficient condition for Bayes-Nash incentive compatibility. Using the lemma from the foregoing section it follows that weak monotonicity is a necessary and sufficient condition for Bayes-Nash incentive compatibility for the case that agents' valuation functions satisfy non-decreasing expected differences. The auction settings considered by Myerson [11] and Malakhov \& Vohra [10] are special cases of this framework. Compared to their settings, our one-dimensional framework allows not only for a broader class of type spaces but also for alternative forms of interdependencies between agents valuations. This is illustrated at the end of Section 5. Using the Myerson [11] setting we illustrate how payments can be constructed using the network 
approach.

In Section 5 the case of multi-dimensional types is considered. Agents' type spaces are assumed to be convex and their valuation functions are assumed to be linear w.r.t. to their own true types. Even under these restrictions, weak monotonicity alone is not sufficient for Bayes-Nash incentive compatibility, which is again illustrated by an example. However, we show that weak monotonicity together with an integrability condition is both necessary and sufficient for Bayes-Nash incentive compatibility. Using examples it is illustrated that weak monotonicity and the integrability condition do not imply each other. The setting of a singleitem auction with externalities considered by Jehiel, Moldovanu \& Stacchetti [5] and the social choice setting considered by Jehiel \& Moldovanu [4] are special cases of the framework presented in this section. Compared to their settings, our multi-dimensional framework allows for a broader class of possible interdependencies between agents valuations. This is illustrated at the end of the section.

The main contribution of this paper is thus to derive for the settings described above a complete characterization of Bayes-Nash incentive compatibility in terms of weak monotonicity and, where necessary, additional properties. Thereby we achieve characterizations that depend purely on the valuations and the allocation rule. The characterizations resemble the ones derived by Rochet [12] for dominant strategy incentive compatibility. However, our results do not follow from Rochet immediately, as we cover broader classes of type spaces and interdependent valuations.

\section{The Model \& Basic Definitions}

There is a set of agents $N=\{1, \ldots, n\}$. Each agent $i$ has a type $t^{i} \in T^{i}$ with $T^{i} \subseteq \mathbb{R}^{k}$. $T$ denotes the set of all type profiles $t=\left(t^{1}, \ldots, t^{n}\right)$, and $T^{-i}$ denotes the set of all type profiles $t^{-i}=\left(t^{1}, \ldots, t^{i-1}, t^{i+1}, \ldots, t^{n}\right)$. A payment rule is a function

$$
P: T \mapsto \mathbb{R}^{n},
$$

so given a report profile $r^{-i}$ of the others, reporting a type $r^{i}$ results in a payment $P_{i}\left(r^{i}, r^{-i}\right)$ for agent $i$. Denoting the set of outcomes by $\Gamma$, an allocation rule is a function

$$
f: T \mapsto \Gamma \text {. }
$$


We allow for interdependent valuations across agents, that is, agents' valuations do not only depend on their own types but on the types of all agents. As an example one can think of an auction for a painting (see Klemperer [6]) where agents' types reflect how much they like the painting. An agent's valuation for owning the painting depends on the types of the others as they affect the possible resale value of the painting and the owner's prestige. Take agent $i$ having true type $t^{i}$ and reporting $r^{i}$ while the others have true types $t^{-i}$ and report $r^{-i}$. The value that agent $i$ assigns to the resulting allocation is denoted by $v^{i}\left(f\left(r^{i}, r^{-i}\right) \mid t^{i}, t^{-i}\right)$. Utilities are quasi-linear, that is, an agent's utility is his valuation of an allocation minus his payment.

Agents' types are independently distributed. Let $\pi^{i}$ denote the density on $T^{i}$. The joint density $\pi^{-i}$ on $T^{-i}$ is then given by

$$
\pi^{-i}\left(t^{-i}\right)=\prod_{\substack{j \in N \\ j \neq i}} \pi^{j}\left(t^{j}\right) .
$$

Assume that agent $i$ believes all other agents to report truthfully. If agent $i$ has true type $t^{i}$, then his expected utility ${ }^{1}$ for making a report $r^{i}$ is given by

$$
\begin{aligned}
U^{i}\left(r^{i} \mid t^{i}\right) & =\int_{T^{-i}}\left(v^{i}\left(f\left(r^{i}, t^{-i}\right) \mid t^{i}, t^{-i}\right)-P_{i}\left(r^{i}, t^{-i}\right)\right) \pi^{-i}\left(t^{-i}\right) d t^{-i} \\
& =E_{-i}\left[v^{i}\left(f\left(r^{i}, t^{-i}\right) \mid t^{i}, t^{-i}\right)-P_{i}\left(r^{i}, t^{-i}\right)\right]
\end{aligned}
$$

We assume $E_{-i}\left[v^{i}\left(f\left(r^{i}, t^{-i}\right) \mid t^{i}, t^{-i}\right)\right]$ to be finite $\forall r^{i}, t^{i} \in T^{i}$.

An allocation rule $f$ is Bayes-Nash incentive compatible if there exists a payment rule $P$ such that $\forall i \in N$ and $\forall r^{i}, \tilde{r}^{i} \in T^{i}$ :

$$
E_{-i}\left[v^{i}\left(f\left(r^{i}, t^{-i}\right) \mid r^{i}, t^{-i}\right)-P_{i}\left(r^{i}, t^{-i}\right)\right] \geq E_{-i}\left[v^{i}\left(f\left(\tilde{r}^{i}, t^{-i}\right) \mid r^{i}, t^{-i}\right)-P_{i}\left(\tilde{r}^{i}, t^{-i}\right)\right]
$$

Symmetrically, we have also

$$
E_{-i}\left[v^{i}\left(f\left(\tilde{r}^{i}, t^{-i}\right) \mid \tilde{r}^{i}, t^{-i}\right)-P_{i}\left(\tilde{r}^{i}, t^{-i}\right)\right] \geq E_{-i}\left[v^{i}\left(f\left(r^{i}, t^{-i}\right) \mid \tilde{r}^{i}, t^{-i}\right)-P_{i}\left(r^{i}, t^{-i}\right)\right] .
$$

By adding (2) and (3) we get the following monotonicity condition: ${ }^{2}$

\footnotetext{
${ }^{1}$ The definition of expected utility is only given for the continuous case. However, in Section 4 we also allow for discrete type spaces. In the discrete case the integral is replaced by a sum.

${ }^{2}$ Expected payments cancel since we work under the assumption of independently distributed types.
} 
Definition 1 (Weak Monotonicity) An allocation rule $f$ satisfies weak monotonicity if $\forall i \in N$ and $\forall r^{i}, \tilde{r}^{i} \in T^{i}$ :

$$
\begin{aligned}
& E_{-i}\left[v^{i}\left(f\left(r^{i}, t^{-i}\right) \mid r^{i}, t^{-i}\right)-v^{i}\left(f\left(\tilde{r}^{i}, t^{-i}\right) \mid r^{i}, t^{-i}\right)\right] \\
\geq & E_{-i}\left[v^{i}\left(f\left(r^{i}, t^{-i}\right) \mid \tilde{r}^{i}, t^{-i}\right)-v^{i}\left(f\left(\tilde{r}^{i}, t^{-i}\right) \mid \tilde{r}^{i}, t^{-i}\right)\right] .
\end{aligned}
$$

This condition is the expected utility equivalent to the weak monotonicity (W-MON) condition of Lavi, Mu'alem \& Nisan [8], the non-decreasing in marginal utility condition (NDMU) of Bikhchandani, Chatterji \& Sen [2] and the 2-cycle inequality of Gui, Müller \& Vohra [3]. Obviously, weak monotonicity is a necessary condition for Bayes-Nash incentive compatibility. In the following sections we present settings where weak monotonicity is also a sufficient condition or where it is sufficient together with an integrability condition.

Furthermore, let us introduce the following condition for agents' valuation functions:

Definition 2 (Non-decreasing Expected Differences) Take $r^{i}, \tilde{r}^{i}, t^{i}, \tilde{t}^{i} \in T^{i}$ such that

$$
\begin{aligned}
& E_{-i}\left[v^{i}\left(f\left(r^{i}, t^{-i}\right) \mid t^{i}, t^{-i}\right)-v^{i}\left(f\left(\tilde{r}^{i}, t^{-i}\right) \mid t^{i}, t^{-i}\right)\right] \\
\geq & E_{-i}\left[v^{i}\left(f\left(r^{i}, t^{-i}\right) \mid \tilde{t}^{i}, t^{-i}\right)-v^{i}\left(f\left(\tilde{r}^{i}, t^{-i}\right) \mid \tilde{t}^{i}, t^{-i}\right)\right] .
\end{aligned}
$$

The valuation function satisfies non-decreasing expected differences if $\forall \bar{t}^{i} \in T^{i}$ s.t. $\bar{t}^{i}=(1-\alpha) \tilde{t}^{i}+\alpha t^{i}, \alpha>1$ we have

$$
\begin{aligned}
& E_{-i}\left[v^{i}\left(f\left(r^{i}, t^{-i}\right) \mid \bar{t}^{i}, t^{-i}\right)-v^{i}\left(f\left(\tilde{r}^{i}, t^{-i}\right) \mid \bar{t}^{i}, t^{-i}\right)\right] \\
\geq & E_{-i}\left[v^{i}\left(f\left(r^{i}, t^{-i}\right) \mid t^{i}, t^{-i}\right)-v^{i}\left(f\left(\tilde{r}^{i}, t^{-i}\right) \mid t^{i}, t^{-i}\right)\right] .
\end{aligned}
$$

This condition deals with the marginal change in expected valuation with respect to the report. Consider the change in expected valuation for making a report $r^{i}$ instead of $\tilde{r}^{i}$. Assume that there exist types $t^{i}$ and $\tilde{t}^{i}$ such that this change is larger or at least as large if the agent has true type $t^{i}$ instead of $\tilde{t}^{i}$. Now consider the agent having a true type which is even further away from $\tilde{t}^{i}$ than $t^{i}$ (in the direction of $t^{i}$ ). The condition then requires that the change in expected valuation is at least as large as in the case where the agent has true type $t^{i}$. This requirement is comparable to the condition known as increasing (or isotone) differences which asserts that the marginal change in valuation with respect to the allocation is increasing in the type. 


\section{A Network Interpretation}

We begin this section by briefly reviewing a well-known result from the field of network flow theory. ${ }^{3}$ Let $X=\left\{x_{1}, \ldots, x_{k}\right\}$ be a finite set of variables. Consider the following system of constraints:

$$
x_{i}-x_{j} \leq w_{i j} \quad \forall i, j \in\{1, \ldots, k\}
$$

where $w_{i j}$ is some constant specific to the ordered pair $(i, j)$. The system can be associated with a network by constructing a directed, weighted graph whose nodes correspond to the variables. A directed edge is put between each ordered pair of nodes. The length of the edge from the node corresponding to $x_{i}$ to the node corresponding to $x_{j}$ is given by $w_{i j}$.

It is a well-known result (see e.g. Shostak [16]) that the system of linear inequalities in (4) is feasible, that is, there exists an assignment of real values to the variables such that the constraints in (4) are satisfied, if and only if there is no negative length cycle in the associated network. Furthermore, if the system is feasible then one feasible solution is to assign to each $x_{i}$ the length of a shortest path from some arbitrary source node to the node associated with $x_{i}$.

In order to see that the constraints in (2) have a natural network interpretation it is useful to rewrite (2) as follows:

$$
E_{-i}\left[P_{i}\left(r^{i}, t^{-i}\right)-P_{i}\left(\tilde{r}^{i}, t^{-i}\right)\right] \leq E_{-i}\left[v^{i}\left(f\left(r^{i}, t^{-i}\right) \mid r^{i}, t^{-i}\right)-v^{i}\left(f\left(\tilde{r}^{i}, t^{-i}\right) \mid r^{i}, t^{-i}\right)\right] .
$$

Considering a specific allocation rule, the right-hand side of (5) is a constant. Thus, we have a system of difference constraints as described in (4) (except that we are now dealing with a potentially infinite number of variables).

Given this observation, we associate the system of inequalities with a network in the same way as is described above. For each agent we build a complete directed graph $T_{f}^{i}$. A node is associated with each type and a directed edge is put between each ordered pair of nodes. For agent $i$ the length of an edge directed from $r^{i}$ to $\tilde{r}^{i}$ is denoted $l^{i}\left(r^{i}, \tilde{r}^{i}\right)$ and is defined as the cost of manipulation:

$$
l^{i}\left(r^{i}, \tilde{r}^{i}\right)=E_{-i}\left[v^{i}\left(f\left(r^{i}, t^{-i}\right) \mid r^{i}, t^{-i}\right)-v^{i}\left(f\left(\tilde{r}^{i}, t^{-i}\right) \mid r^{i}, t^{-i}\right)\right] .
$$

\footnotetext{
${ }^{3}$ A comprehensive introduction to network flows can be found in Ahuja, Magnanti \& Orlin [1].
} 
For technical reasons we allow for loops. However, note that an edge directed from $r^{i}$ to $r^{i}$ has length $l^{i}\left(r^{i}, r^{i}\right)=0$.

Using this definition of the edge lengths, the weak monotonicity condition can be written as

$$
l^{i}\left(r^{i}, \tilde{r}^{i}\right)+l^{i}\left(\tilde{r}^{i}, r^{i}\right) \geq 0 \quad \forall i \in N, \forall r^{i}, \tilde{r}^{i} \in T^{i} .
$$

So weak monotonicity corresponds to the absence of negative length 2-cycles in the graphs described above.

Rochet [12] observed that dominant strategy incentive compatibility can be characterized in terms of the absence of finite, negative length cycles in similar graphs. Using the same proof technique, we can derive such a characterization for Bayes-Nash incentive compatibility as well.

Theorem 1 An allocation rule $f$ is Bayes-Nash incentive compatible if and only if there is no finite, negative length cycle in $T_{f}^{i}, \forall i \in N$.

Proof (Adapted from Rochet [12].)

Take some agent $i$ and let $C=\left\{r_{1}^{i}, \ldots, r_{m}^{i}, r_{m+1}^{i}=r_{1}^{i}\right\}$ denote a finite cycle in $T_{f}^{i}$. Let us assume that $f$ is Bayes-Nash incentive compatible. This implies, using (5) and the edge length definition (6), that for every $j \in\{1, \ldots, m\}$,

$$
E_{-i}\left[P_{i}\left(r_{j}^{i}, t^{-i}\right)-P_{i}\left(r_{j+1}^{i}, t^{-i}\right)\right] \leq l^{i}\left(r_{j}^{i}, r_{j+1}^{i}\right) .
$$

Adding up these inequalities yields

$$
0 \leq \sum_{j=1}^{m} l^{i}\left(r_{j}^{i}, r_{j+1}^{i}\right),
$$

so $C$ has non-negative length.

Conversely, let us assume that there exists no finite, negative length cycle in $T_{f}^{i}, \forall i \in N$. For each agent $i$ we pick an arbitrary source node $r_{0}^{i} \in T^{i}$ and define $\forall r^{i} \in T^{i}$

$$
p^{i}\left(r^{i}\right)=\inf \sum_{j=1}^{m} l^{i}\left(r_{j}^{i}, r_{j+1}^{i}\right),
$$

where the infimum is taken over all finite paths $A=\left\{r_{1}^{i}=r^{i}, \ldots, r_{m+1}^{i}=r_{0}^{i}\right\}$ in $T_{f}^{i}$, that is, all finite paths that start at $r^{i}$ and end at $r_{0}^{i}$. Absence of finite, negative length cycles implies that $p^{i}\left(r_{0}^{i}\right)=0$. Furthermore, $\forall r^{i} \in T^{i}$ we have

$$
p^{i}\left(r_{0}^{i}\right) \leq p^{i}\left(r^{i}\right)+l^{i}\left(r_{0}^{i}, r^{i}\right)
$$


which implies that $p^{i}\left(r^{i}\right)$ is finite. For every pair $r^{i}, \tilde{r}^{i} \in T^{i}$ we also have

$$
p^{i}\left(r^{i}\right) \leq p^{i}\left(\tilde{r}^{i}\right)+l^{i}\left(r^{i}, \tilde{r}^{i}\right)
$$

Thus, by setting ${ }^{4} P_{i}\left(r^{i}, t^{-i}\right)=p^{i}\left(r^{i}\right), \forall t^{-i} \in T^{-i}$, and using (6) we get

$$
E_{-i}\left[P_{i}\left(r^{i}, t^{-i}\right)-P_{i}\left(\tilde{r}^{i}, t^{-i}\right)\right] \leq E_{-i}\left[v^{i}\left(f\left(r^{i}, t^{-i}\right) \mid r^{i}, t^{-i}\right)-v^{i}\left(f\left(\tilde{r}^{i}, t^{-i}\right) \mid r^{i}, t^{-i}\right)\right] .
$$

Hence, the constraints in (5) are satisfied and $f$ is Bayes-Nash incentive compatible.

Let us conclude this section with a condition for the costs of manipulation that is used in the derivation of the characterization theorems presented in the following sections.

Definition 3 (Decomposition Monotonicity) The costs of manipulation are decomposition monotone if $\forall \underline{r}^{i}, \bar{r}^{i} \in T^{i}$ and $\forall r^{i} \in T^{i}$ s.t. $r^{i}=(1-\alpha) \underline{r}^{i}+\alpha \bar{r}^{i}, \alpha \in(0,1)$ we have

$$
l^{i}\left(\underline{r}^{i}, \bar{r}^{i}\right) \geq l^{i}\left(\underline{r}^{i}, r^{i}\right)+l^{i}\left(r^{i}, \bar{r}^{i}\right) .
$$

So looking at a pair of nodes, if decomposition monotonicity holds then the direct edge between those nodes is at least as long as any path connecting the same two nodes via nodes lying on the line segment between them. Figure 1 gives an illustrative example. Decomposition monotonicity implies that the edge from $\underline{r}^{i}$ to $\bar{r}^{i}$ is at least as long as the path $\left.A=\left\{\underline{r}^{i}, r_{* *}^{i}, \bar{r}^{i}\right\}\right\}$ and that $A$ is at least as long as the path $\left.\tilde{A}=\left\{\underline{r}^{i}, r_{*}^{i}, r_{* *}^{i}, r_{* * *}^{i}, \bar{r}^{i}\right\}\right\}$.

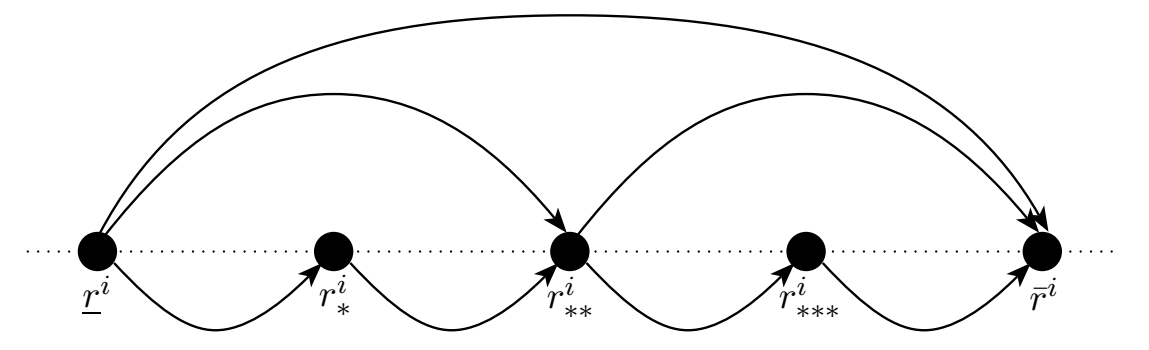

Figure 1: Decomposition monotonicity.

Weak monotonicity and non-decreasing expected differences together imply that the costs of manipulation are decomposition monotone:

\footnotetext{
${ }^{4}$ Note that it is sufficient to set $E_{-i}\left[P_{i}\left(r^{i}, t^{-i}\right)\right]=p^{i}\left(r^{i}\right)+c$. This allows for a variety of payment rules yielding the same expected payments up to an additive constant.
} 
Lemma 1 If $f$ satisfies weak monotonicity and the valuation function satisfies non-decreasing expected differences then the costs of manipulation are decomposition monotone.

\section{Proof}

Take some agent $i$ and let $\underline{r}^{i}, \bar{r}^{i} \in T^{i}$. Let $r^{i} \in T^{i}$ such that $r^{i}=(1-\alpha) \underline{r}^{i}+\alpha \bar{r}^{i}$ for some $\alpha \in(0,1)$. Weak monotonicity implies that

$$
\begin{aligned}
& E_{-i}\left[v^{i}\left(f\left(r^{i}, t^{-i}\right) \mid r^{i}, t^{-i}\right)-v^{i}\left(f\left(\bar{r}^{i}, t^{-i}\right) \mid r^{i}, t^{-i}\right)\right] \\
\geq & E_{-i}\left[v^{i}\left(f\left(r^{i}, t^{-i}\right) \mid \bar{r}^{i}, t^{-i}\right)-v^{i}\left(f\left(\bar{r}^{i}, t^{-i}\right) \mid \bar{r}^{i}, t^{-i}\right)\right] .
\end{aligned}
$$

Since the valuation function satisfies non-decreasing expected differences we have

$$
\begin{aligned}
& E_{-i}\left[v^{i}\left(f\left(r^{i}, t^{-i}\right) \mid \underline{r}^{i}, t^{-i}\right)-v^{i}\left(f\left(\bar{r}^{i}, t^{-i}\right) \mid \underline{r}^{i}, t^{-i}\right)\right] \\
\geq & E_{-i}\left[v^{i}\left(f\left(r^{i}, t^{-i}\right) \mid r^{i}, t^{-i}\right)-v^{i}\left(f\left(\bar{r}^{i}, t^{-i}\right) \mid r^{i}, t^{-i}\right)\right] .
\end{aligned}
$$

Adding $E_{-i}\left[v^{i}\left(f\left(\underline{r}^{i}, t^{-i}\right) \mid \underline{r}^{i}, t^{-i}\right)-v^{i}\left(f\left(r^{i}, t^{-i}\right) \mid \underline{r}^{i}, t^{-i}\right)\right]$ on both sides of the later inequality yields

$$
\begin{array}{r}
E_{-i}\left[v^{i}\left(f\left(r^{i}, t^{-i}\right) \mid \underline{r}^{i}, t^{-i}\right)-v^{i}\left(f\left(\bar{r}^{i}, t^{-i}\right) \mid \underline{r}^{i}, t^{-i}\right)\right] \\
+E_{-i}\left[v^{i}\left(f\left(\underline{r}^{i}, t^{-i}\right) \mid \underline{r}^{i}, t^{-i}\right)-v^{i}\left(f\left(r^{i}, t^{-i}\right) \mid \underline{r}^{i}, t^{-i}\right)\right] \\
\geq \quad E_{-i}\left[v^{i}\left(f\left(r^{i}, t^{-i}\right) \mid r^{i}, t^{-i}\right)-v^{i}\left(f\left(\bar{r}^{i}, t^{-i}\right) \mid r^{i}, t^{-i}\right)\right] \\
+E_{-i}\left[v^{i}\left(f\left(\underline{r}^{i}, t^{-i}\right) \mid \underline{r}^{i}, t^{-i}\right)-v^{i}\left(f\left(r^{i}, t^{-i}\right) \mid \underline{r}^{i}, t^{-i}\right)\right] .
\end{array}
$$

Notice that the first and the last term on the left-hand side of the inequality cancel, hence

$$
\begin{aligned}
& E_{-i}\left[v^{i}\left(f\left(\underline{r}^{i}, t^{-i}\right) \mid \underline{r}^{i}, t^{-i}\right)-v^{i}\left(f\left(\bar{r}^{i}, t^{-i}\right) \mid \underline{r}^{i}, t^{-i}\right)\right] \\
\geq \quad & E_{-i}\left[v^{i}\left(f\left(\underline{r}^{i}, t^{-i}\right) \mid \underline{r}^{i}, t^{-i}\right)-v^{i}\left(f\left(r^{i}, t^{-i}\right) \mid \underline{r}^{i}, t^{-i}\right)\right] \\
+ & E_{-i}\left[v^{i}\left(f\left(r^{i}, t^{-i}\right) \mid r^{i}, t^{-i}\right)-v^{i}\left(f\left(\bar{r}^{i}, t^{-i}\right) \mid r^{i}, t^{-i}\right)\right] .
\end{aligned}
$$

Using (6) this can be written as

$$
l^{i}\left(\underline{r}^{i}, \bar{r}^{i}\right) \geq l^{i}\left(\underline{r}^{i}, r^{i}\right)+l^{i}\left(r^{i}, \bar{r}^{i}\right)
$$

so the costs of manipulation are decomposition monotone. 


\section{One-Dimensional Types}

In this section we consider the one-dimensional case where $T^{i} \subseteq \mathbb{R}$. For this setting we can show that weak monotonicity is not only a necessary but also a sufficient condition for Bayes-Nash incentive compatibility if decomposition monotonicity is satisfied.

Theorem 2 Suppose that the allocation rule $f$ and agents' valuation functions are such that the costs of manipulation satisfy decomposition monotonicity. Then, $f$ is Bayes-Nash incentive compatible if and only if $f$ satisfies weak monotonicity.

\section{Proof}

As mentioned in Section 2, the necessity of weak monotonicity follows trivially. For the other direction note that weak monotonicity corresponds to the absence of negative length 2-cycles in $T_{f}^{i}, \forall i \in N$ (see Section 3). In order to establish sufficiency, we show that this implies that there does not exist any finite cycle with negative length. Bayes-Nash incentive compatibility then follows from Theorem 1.

Take some agent $i$ and let $C=\left\{r_{1}^{i}, \ldots, r_{m}^{i}, r_{m+1}^{i}=r_{1}^{i}\right\}$ denote a finite cycle in $T_{f}^{i}$. Whenever an edge of $C$ connects two non-neighboring nodes, we substitute this edge with a path connecting the same two nodes via edges that have the same direction and only connect neighboring nodes. By doing this we generate a new cycle $\tilde{C}$ that has the same nodes as $C$ but consists only of edges between neighboring nodes, see for example Figure 2.

Decomposition monotonicity implies that the length of $C$ is larger than or equal to the length of $\tilde{C}$. Since $\tilde{C}$ is a cycle, we know that at each node the number of edges entering equals the number of edges leaving. This implies that the length of $\tilde{C}$ can be written as the sum of 2-cycle lengths. Since there are no negative length 2-cycles, it follows that $C$ has non-negative length.
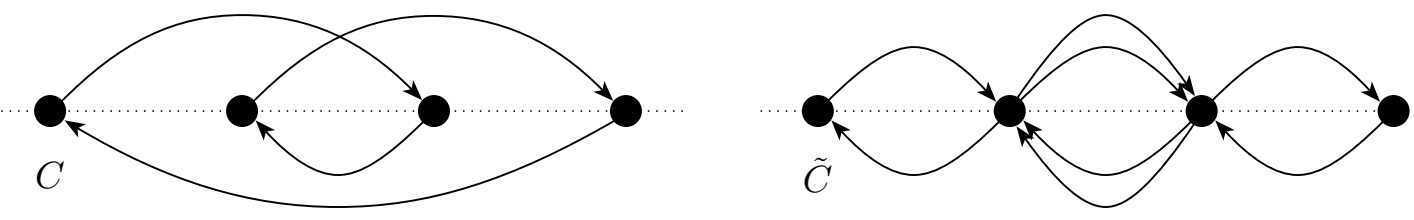

Figure 2: The original cycle $C$ and the newly generated cycle $\tilde{C}$. 
If agents' valuation functions satisfy non-decreasing expected differences we can state directly the following:

Theorem 3 Suppose that agents' valuation functions satisfy non-decreasing expected differences. Then, the allocation rule $f$ is Bayes-Nash incentive compatible if and only if $f$ satisfies weak monotonicity.

\section{Proof}

Again, the necessity of weak monotonicity follows trivially (see Section 2). In order to establish sufficiency, note that weak monotonicity together with non-decreasing expected differences implies that the costs of manipulation are decomposition monotone, see Lemma 1. Finally apply Theorem 2 .

Note that weak monotonicity might not be sufficient for Bayes-Nash incentive compatibility if the cost of manipulation are not decomposition monotone, as we illustrate with the following example:

Example 1 For simplicity we assume that there exists only a single agent. His type space $T=\{x, y, z\}$ consists of three types $x, y, z \in \mathbb{R}$, for which we assume $x<y<z$. There are three possible outcomes, specifically $\Gamma=\{a, b, c\}$. The agent values the different outcomes, depending on his type, according to the following valuation matrix $V$ :

\begin{tabular}{c|c|c|c|}
\multicolumn{1}{c}{$x$} & $y$ & \multicolumn{1}{c}{} \\
\multicolumn{1}{c}{$a$} \\
\cline { 2 - 4 }$a$ & 2 & 0 & 3 \\
\cline { 2 - 4 }$b$ & 3 & 2 & 0 \\
\cline { 2 - 4 }$c$ & 0 & 3 & 2 \\
\hline
\end{tabular}

The allocation rule $f$ is defined as follows: $f(x)=a, f(y)=b, f(z)=c$. So if the agent reports truthfully his type, the allocation rule assigns his second most preferred outcome. The corresponding network $T_{f}$ is depicted in Figure 3.

Decomposition monotonicity is not satisfied since $l(z, x)<l(z, y)+l(y, x)$. As easily can be checked, all 2-cycles have length 1, so weak monotonicity is satisfied. However, the 3-cycle $C=\{x, y, z, x\}$ has length $l(x, y)+l(y, z)+l(z, x)=-3$. The existence of such a negative length cycle implies that $f$ is not Bayes-Nash incentive compatible (see Theorem 1). 


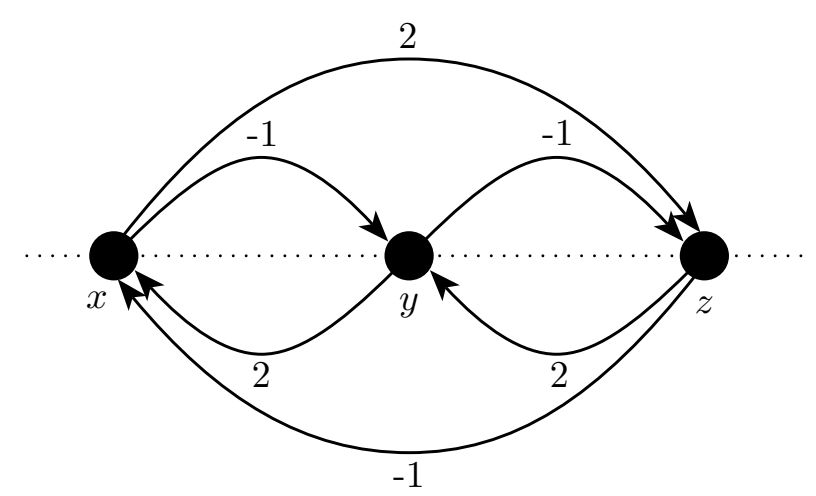

Figure 3: The network $T_{f}$ in Example 1.

\subsection{Application to Single-item Auctions}

As a special case let us consider a single-item auction. Specifically, we look at a setting introduced by Myerson [11]. In this setting a single, indivisible object is auctioned off to one of several agents. An agent's type reflects his initial value estimate for the object. We assume that $T^{i}=\left[a^{i}, b^{i}\right]$ with $-\infty<a^{i}<b^{i}<\infty$. Given reports from all agents, the allocation rule $f: T \mapsto[0,1]^{n}$ assigns to each agent a probability for winning the object. So the outcome set $\Gamma$ is the set of all possible winning probability profiles. Agent $i$ 's probability to win, given a report profile $t \in T$, is denoted $f^{i}(t)$. The allocation rule must satisfy the probability conditions $\sum_{i=1}^{n} f^{i}(t) \leq 1$ and $0 \leq f^{i}(t) \leq 1, \forall i \in N, \forall t \in T$.

Agents' valuations are assumed to be interdependent. If an agent would get to know the value estimate of some other agent, he would want to revise his own initial value estimate for the object. For instance, the object is a painting (see also example in Section 2) and the agent is uncertain about whether he is dealing with an original or a forgery. Learning that another agent has a low value estimate, suggesting a tendency towards forgery, would incline him to revise his own value estimate downwards. We assume that agents make these revisions additively according to $n$ revision effect functions $e^{j}: T^{j} \mapsto \mathbb{R}, j \in N$ : if agent $i$ learns that agent $j$ has type $t^{j}$, he revises his initial value estimate by adding $e^{j}\left(t^{j}\right)$ to it. ${ }^{5}$ In order

\footnotetext{
${ }^{5}$ Modelling the valuation interdependencies in this way implies that actually all agents $i \neq j$ would revise their initial estimates by the same amount $e^{j}\left(t^{j}\right)$. Without affecting any of the results, one could also allow for agent specific adjustments by assuming that each agent $i$ has $n-1$ revision effect functions $e_{i}^{j}: T^{j} \mapsto \mathbb{R}$, $j \in N, j \neq i$.
} 
to justify that an agent's type reflects his initial value estimate for the object, we assume in addition that

$$
\int_{T^{j}} e^{j}\left(t^{j}\right) \pi^{j}\left(t^{j}\right) d t^{j}=0
$$

that is, revision effects have an expected value of zero. ${ }^{6}$

Take agent $i$ having true type $t^{i}$ and reporting $r^{i}$ while the others have true types $t^{-i}$ and reports $r^{-i}$. The value that this agent assigns to the resulting allocation is

$$
v^{i}\left(f\left(r^{i}, r^{-i}\right) \mid t^{i}, t^{-i}\right)=\left(t^{i}+\sum_{\substack{j \in N \\ j \neq i}} e^{j}\left(t^{j}\right)\right) f^{i}\left(r^{i}, r^{-i}\right)
$$

Assuming that agent $i$ believes that the others report truthfully, his expected conditional probability to win the object if reporting $r^{i}$ is

$$
q^{i}\left(r^{i}\right)=\int_{T^{-i}} f^{i}\left(r^{i}, t^{-i}\right) \pi^{-i}\left(t^{-i}\right) d t^{-i}
$$

and his expected valuation is

$$
E_{-i}\left[v^{i}\left(f\left(r^{i}, t^{-i}\right) \mid t^{i}, t^{-i}\right)\right]=t^{i} q^{i}\left(r^{i}\right)+\int_{T^{-i}}\left(\sum_{\substack{j \in N \\ j \neq i}} e^{j}\left(t^{j}\right)\right) f^{i}\left(r^{i}, t^{-i}\right) \pi^{-i}\left(t^{-i}\right) d t^{-i}
$$

Using (8), the weak monotonicity condition becomes

$$
\left(r^{i}-\tilde{r}^{i}\right)\left(q^{i}\left(r^{i}\right)-q^{i}\left(\tilde{r}^{i}\right)\right) \geq 0 \quad \forall i \in N, \forall r^{i}, \tilde{r}^{i} \in T^{i}
$$

Myerson [11] shows that a mechanism $(f, P)$ is Bayes-Nash incentive compatible if and only if (9) is satisfied and

$$
U^{i}\left(r^{i} \mid r^{i}\right)=U^{i}\left(a^{i} \mid a^{i}\right)+\int_{a^{i}}^{r^{i}} q^{i}(s) d s \quad \forall i \in N, \forall r^{i} \in T^{i}
$$

where $U^{i}\left(r^{i} \mid r^{i}\right)$ denotes agent $i$ 's expected utility (see (1) for definition) for truthfully reporting $r^{i}$.

As one can easily verify, agents' valuation functions in this single-item auction setting satisfy non-decreasing expected differences. Thus, we can directly apply the results derived earlier in this section. From Theorem 3 it follows that $f$ is Bayes-Nash incentive compatible if and only if the weak monotonicity condition in (9) is satisfied.

\footnotetext{
${ }^{6}$ The stated results do not depend on this assumption. However, without it, the interpretation of agents' types would change.
} 
As described in the proof of Theorem 1, corresponding payments can be constructed by using shortest path lengths. For each $i \in N$, let us pick $a^{i}$ as the source node in $T_{f}^{i}$. Thus, if agent $i$ reports $r^{i}$, he has to make a payment

$$
P_{i}\left(r^{i}\right)=\inf \sum_{j=1}^{m} l^{i}\left(r_{j}^{i}, r_{j+1}^{i}\right),
$$

where the infimum is taken over all finite paths $A=\left\{r_{1}^{i}=r^{i}, \ldots, r_{m+1}^{i}=a^{i}\right\}$ in $T_{f}^{i}$, that is, all finite paths from $r^{i}$ to $a^{i}$. Considering the length of such a finite path, rewriting yields

$$
\begin{aligned}
\sum_{j=1}^{m} l^{i}\left(r_{j}^{i}, r_{j+1}^{i}\right)= & \sum_{j=1}^{m} E_{-i}\left[v^{i}\left(f\left(r_{j}^{i}, t^{-i}\right) \mid r_{j}^{i}, t^{-i}\right)-v^{i}\left(f\left(r_{j+1}^{i}, t^{-i}\right) \mid r_{j}^{i}, t^{-i}\right)\right] \\
= & E_{-i}\left[v^{i}\left(f\left(r_{1}^{i}, t^{-i}\right) \mid r_{1}^{i}, t^{-i}\right)-v^{i}\left(f\left(r_{m+1}^{i}, t^{-i}\right) \mid r_{m}^{i}, t^{-i}\right)\right] \\
& -\sum_{j=1}^{m-1} E_{-i}\left[v^{i}\left(f\left(r_{j+1}^{i}, t^{-i}\right) \mid r_{j}^{i}, t^{-i}\right)-v^{i}\left(f\left(r_{j+1}^{i}, t^{-i}\right) \mid r_{j+1}^{i}, t^{-i}\right)\right] \\
= & E_{-i}\left[v^{i}\left(f\left(r_{1}^{i}, t^{-i}\right) \mid r_{1}^{i}, t^{-i}\right)-v^{i}\left(f\left(r_{m+1}^{i}, t^{-i}\right) \mid r_{m+1}^{i}, t^{-i}\right)\right] \\
& -\sum_{j=1}^{m} E_{-i}\left[v^{i}\left(f\left(r_{j+1}^{i}, t^{-i}\right) \mid r_{j}^{i}, t^{-i}\right)-v^{i}\left(f\left(r_{j+1}^{i}, t^{-i}\right) \mid r_{j+1}^{i}, t^{-i}\right)\right] \\
= & E_{-i}\left[v^{i}\left(f\left(r^{i}, t^{-i}\right) \mid r^{i}, t^{-i}\right)-v^{i}\left(f^{i}\left(a^{i}, t^{-i}\right) \mid a^{i}, t^{-i}\right)\right] \\
& -\sum_{j=1}^{m}\left(r_{j}^{i}-r_{j+1}^{i}\right) q^{i}\left(r_{j+1}^{i}\right) .
\end{aligned}
$$

The first equality follows from the definition of the edge length given in (6). The second equality follows from rearranging the terms of the summation. The third equality is derived by adding and subtracting $E_{-i}\left[v^{i}\left(f\left(r_{m+1}^{i}, t^{-i}\right) \mid r_{m+1}^{i}, t^{-i}\right)\right]$. To derive the last equality we use (8) and that $r_{1}^{i}=r^{i}, r_{m+1}^{i}=a^{i}$.

Weak monotonicity implies that $q^{i}\left(r^{i}\right)$ is Riemann integrable. ${ }^{7}$ Furthermore, since the valuation function satisfies non-decreasing expected differences, decomposition monotonicity is satisfied (see Lemma 1). So considering any finite path $A$ in $T_{f}^{i}$ connecting $r^{i}$ and $a^{i}$, we can construct paths that are shorter (or as long) by letting them visit the same nodes as $A$ and also additional nodes inbetween (see also example in Figure 1). In the limit, as $m \rightarrow \infty$, the distance between neighboring nodes goes to zero and

$$
\sum_{j=1}^{m}\left(r_{j}^{i}-r_{j+1}^{i}\right) q^{i}\left(r_{j+1}^{i}\right) \rightarrow \int_{a^{i}}^{r^{i}} q^{i}(s) d s .
$$

\footnotetext{
${ }^{7}$ This result can be found in any advanced analysis textbook, e.g. Maak [9].
} 
Applying the above to (10) yields

$$
P_{i}\left(r^{i}\right)=E_{-i}\left[v^{i}\left(f\left(r^{i}, t^{-i}\right) \mid r^{i}, t^{-i}\right)-v^{i}\left(f^{i}\left(a^{i}, t^{-i}\right) \mid a^{i}, t^{-i}\right)\right]-\int_{a^{i}}^{r^{i}} q^{i}(s) d s,
$$

implying that the expected utility for truthfully reporting $r^{i}$ is 8

$$
U^{i}\left(r^{i} \mid r^{i}\right)=U^{i}\left(a^{i} \mid a^{i}\right)+\int_{a^{i}}^{r^{i}} q^{i}(s) d s .
$$

\section{Multi-Dimensional Types}

In this section we consider the multi-dimensional case where $T^{i} \subseteq \mathbb{R}^{k}$. ${ }^{9}$ We assume that $T^{i}$ is convex for each agent $i$. Furthermore, we now assume that an agent's valuation function is linear in his own true type. So if agent $i$ has true type $t^{i}$ and reports $r^{i}$ while the others have true types $t^{-i}$ and reports $r^{-i}$, his valuation for the resulting allocation is

$$
v^{i}\left(f\left(r^{i}, r^{-i}\right) \mid t^{i}, t^{-i}\right)=\alpha^{i}\left(f\left(r^{i}, r^{-i}\right) \mid t^{-i}\right)+\beta^{i}\left(f\left(r^{i}, r^{-i}\right) \mid t^{-i}\right) t^{i} .
$$

Note that $\alpha^{i}: \Gamma \times T^{-i} \mapsto \mathbb{R}$ and $\beta^{i}: \Gamma \times T^{-i} \mapsto \mathbb{R}^{k}$, i.e. $\alpha^{i}$ assigns to every $\left(\gamma, t^{-i}\right) \in \Gamma \times T^{-i}$

a value in $\mathbb{R}$, whereas $\beta^{i}$ assigns to every $\left(\gamma, t^{-i}\right) \in \Gamma \times T^{-i}$ a value in $\mathbb{R}^{k}$. Similarly, assuming he believes all other agents to report truthfully, agent $i$ 's expected valuation for reporting $r^{i}$ while having true type $t^{i}$ is

$$
E_{-i}\left[v^{i}\left(f\left(r^{i}, t^{-i}\right) \mid t^{i}, t^{-i}\right)\right]=E_{-i}\left[\alpha^{i}\left(f\left(r^{i}, t^{-i}\right) \mid t^{-i}\right)\right]+E_{-i}\left[\beta^{i}\left(f\left(r^{i}, t^{-i}\right) \mid t^{-i}\right)\right] t^{i}
$$

Using (14), the weak monotonicity condition becomes

$$
E_{-i}\left[\beta^{i}\left(f\left(r^{i}, t^{-i}\right) \mid t^{-i}\right)-\beta^{i}\left(f\left(\tilde{r}^{i}, t^{-i}\right) \mid t^{-i}\right)\right]\left(r^{i}-\tilde{r}^{i}\right) \geq 0 \quad \forall i \in N, \forall r^{i}, \tilde{r}^{i} \in T^{i} .
$$

One can easily verify that the linear valuation function described above satisfies nondecreasing expected differences. For this case we showed in Section 4 that weak monotonicity is a sufficient condition for Bayes-Nash incentive compatibility if agents' type spaces are onedimensional (see Theorem 3). Unfortunately, if type spaces are multi-dimensional then weak monotonicity alone is not sufficient anymore, as is illustrated in Example 2.

\footnotetext{
${ }^{8}$ In order to derive (12) one can use that by construction $P_{i}\left(a^{i}\right)=0$ and thus add this term to the right-hand side of (11).

${ }^{9}$ In the special case where agents' type spaces are subsets of lines in $\mathbb{R}^{k}$, the results of the foregoing section go through unchanged.
} 
This example is constructed based on the following insight: Suppose that the allocation function $f$ and the mapping $\beta^{i}$ are such that we can write

$$
E_{-i}\left[\beta^{i}\left(f\left(r^{i}, t^{-i}\right) \mid t^{-i}\right)\right]=r^{i} B_{i}
$$

where $B_{i}$ is some agent specific $k \times k$ matrix. Weak monotonicity requires

$$
\left(r^{i}-\tilde{r}^{i}\right) B_{i}\left(r^{i}-\tilde{r}^{i}\right)^{\prime} \geq 0 \quad \forall r^{i}, \tilde{r}^{i} \in T^{i}
$$

where ' denotes "transposed". Note that

$$
B_{i}=\frac{1}{2}\left(B_{i}+B_{i}^{\prime}\right)+\frac{1}{2}\left(B_{i}-B_{i}^{\prime}\right)
$$

that is, $B_{i}$ can be decomposed into a symmetric part $\frac{1}{2}\left(B_{i}+B_{i}^{\prime}\right)$ and an anti-symmetric part $\frac{1}{2}\left(B_{i}-B_{i}^{\prime}\right)$. Weak monotonicity is already satisfied if the symmetric part of $B_{i}$ is positive semi-definite. However, there are no finite, negative length cycles in $T_{f}^{i}$ (and thus $f$ is BayesNash incentive compatible) if and only if $B_{i}$ is symmetric and positive semi-definite (follows from Rockafellar [14], p.240).

Example 2 For simplicity we assume that there exists only a single agent. His type space $T=\operatorname{conv}\{x, y, z\}$ with generic element $t=\left(t_{x}, t_{y}, t_{z}\right)$ is the convex hull of the three unit vectors in $\mathbb{R}^{3}$, i.e. a simplex with vertices $x=(1,0,0), y=(0,1,0)$ and $z=(0,0,1)$.

There are three elementary outcomes, denoted $a, b$ and $c$. The allocation function $f$ maps every report into a probability distribution over these three outcomes. Thus, the outcome space $\Gamma$ is the set of all possible probability distributions on $\{a, b, c\}$. The generic element $\gamma=$ $\left(\gamma_{a}, \gamma_{b}, \gamma_{c}\right)$ indicates that outcome $a$ is achieved with probability $\gamma_{a}, b$ with probability $\gamma_{b}$ and $c$ with probability $\gamma_{c}$. The elementary outcomes can be associated with the three unit vectors in $\mathbb{R}^{3}$, i.e. $a=(1,0,0), b=(0,1,0)$ and $c=(0,0,1)$. Specifically, we take $f$ to be simply a linear mapping corresponding to the $3 \times 3$ identity matrix $I$. Hence, $f(t)=t I=\left(t_{x}, t_{y}, t_{z}\right)$ and $\Gamma=\operatorname{conv}\{a, b, c\}$. So reporting $t$ results in outcome a being realized with probability $t_{x}, b$ with probability $t_{y}$ and $c$ with probability $t_{z}$. Since there is only one agent, the general valuation function in (14) becomes $v(f(r) \mid t)=\alpha(f(r))+\beta(f(r)) t$. Specifically, we set $\alpha(\gamma)$ equal to zero for all $\gamma \in \Gamma$, and we let $\beta$ be a linear mapping corresponding to the matrix $V$ given in Example 1. Thus, $v(f(r) \mid t)=f(r) V t^{\prime}=r V t^{\prime}$. 
As easily can be checked (by verifying that the symmetric part $\frac{1}{2}\left(V+V^{\prime}\right)$ of $V$ is positive definite), weak monotonicity is satisfied, that is, $(r-\tilde{r}) V(r-\tilde{r})^{\prime} \geq 0, \forall r, \tilde{r} \in T$. Nevertheless, the 3-cycle $C=\{x, y, z, x\}$ has length $l(x, y)+l(y, z)+l(z, x)=-3$ (see also Figure 4). The existence of such a negative length cycle implies that $f$ is not Bayes-Nash incentive compatible (see Theorem 1).

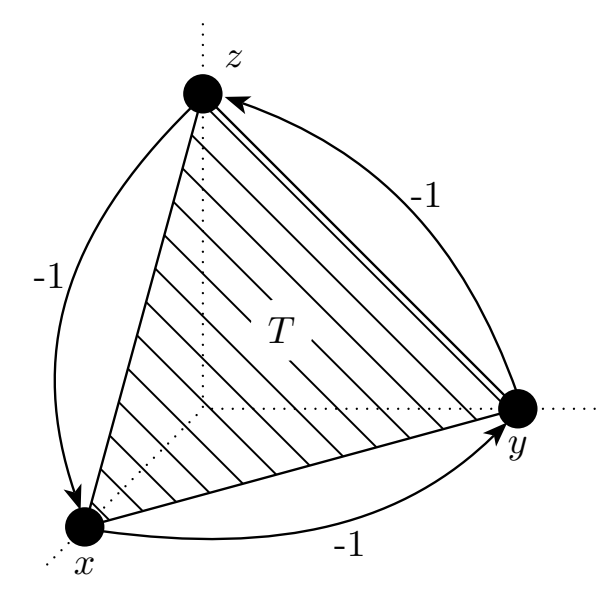

Figure 4: The negative cycle $C$ in Example 2.

From the above example it is evident that weak monotonicity alone is not enough to ensure Bayes-Nash incentive compatibility. However, in the following we are going to show that weak monotonicity together with an integrability condition is sufficient.

Definition 4 (Path Independence) Let $\psi: T^{i} \mapsto \mathbb{R}^{k}$ be a vector field. $\psi$ is called path independent if for any two $\underline{r}^{i}, \bar{r}^{i} \in T^{i}$ the integral of $\psi$ from $\underline{r}^{i}$ to $\bar{r}^{i}$

$$
\int_{\underline{r}^{i}, S}^{\bar{r}^{i}} \psi
$$

is independent of the path of integration $S$.

Note that $E_{-i}\left[\beta^{i}\left(f\left(r^{i}, t^{-i}\right) \mid t^{-i}\right)\right]$ is a vector field $T^{i} \mapsto \mathbb{R}^{k}$.

Theorem 4 Suppose that every agent $i$ has a convex type space and a valuation function which is linear in his true type. Then the following statements are equivalent: 1) $f$ is Bayes-Nash incentive compatible. 
2) $f$ satisfies weak monotonicity and for every agent $i, E_{-i}\left[\beta^{i}\left(f\left(r^{i}, t^{-i}\right) \mid t^{-i}\right)\right]$ is path independent.

\section{Proof}

$(\mathbf{1}) \Rightarrow(\mathbf{2})$ : Let us assume that $f$ is Bayes-Nash incentive compatible. As mentioned in Section 2, the necessity of weak monotonicity follows trivially. Furthermore, from Theorem 1 it follows that for every agent $i$ the graph $T_{f}^{i}$ has no finite, negative length cycles. Let $C=\left\{r_{1}^{i}, \ldots, r_{m}^{i}, r_{m+1}^{i}=r_{1}^{i}\right\}$ denote a finite cycle in $T_{f}^{i}$. Absence of finite, negative length cycles implies that

$$
\sum_{j=1}^{m} l^{i}\left(r_{j}^{i}, r_{j+1}^{i}\right) \geq 0
$$

which can be rewritten using (6) and (14) as

$$
\sum_{j=1}^{m} E_{-i}\left[\beta^{i}\left(f\left(r_{j}^{i}, t^{-i}\right) \mid t^{-i}\right)-\beta^{i}\left(f\left(r_{j+1}^{i}, t^{-i}\right) \mid t^{-i}\right)\right] r_{j}^{i} \geq 0 .
$$

This implies that

$$
\sum_{j=1}^{m} E_{-i}\left[\beta^{i}\left(f\left(r_{j+1}^{i}, t^{-i}\right) \mid t^{-i}\right)\right]\left(r_{j+1}^{i}-r_{j}^{i}\right) \geq 0 .
$$

Thus, $E_{-i}\left[\beta^{i}\left(f\left(r^{i}, t^{-i}\right) \mid t^{-i}\right)\right]$ is cyclically monotone. ${ }^{10}$ From Rockafellar [14], Theorem 24.8 , it follows that there exists a convex function $\varphi: T^{i} \mapsto \mathbb{R}$ such that $E_{-i}\left[\beta^{i}\left(f\left(r^{i}, t^{-i}\right) \mid t^{-i}\right)\right]$ is a selection from its subdifferential mapping, that is,

$$
E_{-i}\left[\beta^{i}\left(f\left(r^{i}, t^{-i}\right) \mid t^{-i}\right)\right] \in \partial \varphi\left(r^{i}\right), \forall r^{i} \in T^{i} .
$$

This implies (see Krishna \& Maenner [7], Theorem 1) that for any smooth path $S$ in $T^{i}$ joining $\underline{r}^{i}$ and $\bar{r}^{i}$ the following holds:

$$
\int_{\underline{r}^{i}, S}^{\bar{r}^{i}} E_{-i}\left[\beta^{i}\left(f\left(r^{i}, t^{-i}\right) \mid t^{-i}\right)\right]=\varphi\left(\bar{r}^{i}\right)-\varphi\left(\underline{r}^{i}\right),
$$

so $E_{-i}\left[\beta^{i}\left(f\left(r^{i}, t^{-i}\right) \mid t^{-i}\right)\right]$ is path independent.

$(\mathbf{2}) \Rightarrow(\mathbf{1})$ : Let us assume that $f$ satisfies weak monotonicity and that for every agent $i$, $E_{-i}\left[\beta^{i}\left(f\left(r^{i}, t^{-i}\right) \mid t^{-i}\right)\right]$ is path independent. Take any edge from $T_{f}^{i}$ and denote its starting node $\underline{r}^{i}$ and its ending node $\bar{r}^{i}$. Let $L$ denote the line segment between $\underline{r}^{i}$ and $\bar{r}^{i}$, i.e. $L=$ $\left\{r^{i} \in T^{i} \mid r^{i}=(1-\alpha) \underline{r}^{i}+\alpha \bar{r}^{i}, \alpha \in[0,1]\right\}$. Now we pick any $r^{i} \in L$ and substitute the original

\footnotetext{
${ }^{10}$ The notion of cyclical monotonicity was introduced by Rockafellar [13].
} 
edge with the path $A=\left\{\underline{r}^{i}, r^{i}, \bar{r}^{i}\right\}$ which has length $l^{i}\left(\underline{r}^{i}, r^{i}\right)+l^{i}\left(r^{i}, \bar{r}^{i}\right)$. As mentioned above, the valuation function satisfies non-decreasing expected differences. Together with weak monotonicity this implies (see Lemma 1):

$$
l^{i}\left(\underline{r}^{i}, \bar{r}^{i}\right) \geq l^{i}\left(\underline{r}^{i}, r^{i}\right)+l^{i}\left(r^{i}, \bar{r}^{i}\right)
$$

that is, the original edge is at least as long as the path $A$. By repeated substitution we can generate a new path $\tilde{A}=\left\{r_{1}^{i}=\underline{r}^{i}, \ldots, r_{m}^{i}, r_{m+1}^{i}=\bar{r}^{i}\right\}$ where $r_{j}^{i} \in L, \forall j \in\{1, \ldots, m+1\}$. Then (1) implies that the original edge is at least as long as $\tilde{A}$, that is,

$$
l^{i}\left(\underline{r}^{i}, \bar{r}^{i}\right) \geq \sum_{j=1}^{m} l^{i}\left(r_{j}^{i}, r_{j+1}^{i}\right),
$$

(see also the example given in Figure 1). Note that ${ }^{11}$

$$
\begin{aligned}
\sum_{j=1}^{m} l^{i}\left(r_{j}^{i}, r_{j+1}^{i}\right)= & E_{-i}\left[v^{i}\left(f\left(\underline{r}^{i}, t^{-i}\right) \mid \underline{r}^{i}, t^{-i}\right)-v^{i}\left(f\left(\bar{r}^{i}, t^{-i}\right) \mid \bar{r}^{i}, t^{-i}\right)\right] \\
+ & \sum_{j=1}^{m} E_{-i}\left[\beta^{i}\left(f\left(r_{j+1}^{i}, t^{-i}\right) \mid t^{-i}\right)\right]\left(r_{j}^{i}-r_{j+1}^{i}\right) .
\end{aligned}
$$

By repeated substitution we can generate paths with more and more edges. In the limit the distance between neighboring nodes goes to zero and

$$
\sum_{j=1}^{m} E_{-i}\left[\beta^{i}\left(f\left(r_{j+1}^{i}, t^{-i}\right) \mid t^{-i}\right)\right]\left(r_{j}^{i}-r_{j+1}^{i}\right) \rightarrow \int_{\underline{r}^{i}, L}^{\bar{r}^{i}} E_{-i}\left[\beta^{i}\left(f\left(r^{i}, t^{-i}\right) \mid t^{-i}\right)\right] .
$$

Thus, the length of $\tilde{A}$ goes to

$$
E_{-i}\left[v^{i}\left(f\left(\underline{r}^{i}, t^{-i}\right) \mid \underline{r}^{i}, t^{-i}\right)-v^{i}\left(f\left(\bar{r}^{i}, t^{-i}\right) \mid \bar{r}^{i}, t^{-i}\right)\right]+\int_{\underline{r}^{i}, L}^{\bar{r}^{i}} E_{-i}\left[\beta^{i}\left(f\left(r^{i}, t^{-i}\right) \mid t^{-i}\right)\right]
$$

as $m \rightarrow \infty$. Now, let $C=\left\{r_{1}^{i}, \ldots, r_{m}^{i}, r_{m+1}^{i}=r_{1}^{i}\right\}$ denote a finite cycle in $T_{f}^{i}$. Furthermore, let $L_{j}$ denote the line segment between $r_{j}^{i}$ and $r_{j+1}^{i}$. The result in (16) and the path independence

\footnotetext{
${ }^{11}$ For a more detailed description of the transformation steps involved, the reader is referred to the single-item auction application in Section 4 .
} 
of $E_{-i}\left[\beta^{i}\left(f\left(r^{i}, t^{-i}\right) \mid t^{-i}\right)\right]$ imply for the length of $C$ that

$$
\begin{aligned}
& \sum_{j=1}^{m} l^{i}\left(r_{j}^{i}, r_{j+1}^{i}\right) \\
\geq & \sum_{j=1}^{m} E_{-i}\left[v^{i}\left(f\left(r_{j}^{i}, t^{-i}\right) \mid r_{j}^{i}, t^{-i}\right)-v^{i}\left(f\left(r_{j+1}^{i}, t^{-i}\right) \mid r_{j+1}^{i}, t^{-i}\right)\right] \\
& +\sum_{j=1}^{m} \int_{r_{j}^{i}, L_{j}}^{r_{j+1}^{i}} E_{-i}\left[\beta^{i}\left(f\left(r^{i}, t^{-i}\right) \mid t^{-i}\right)\right] \\
= & 0
\end{aligned}
$$

that is, $C$ has non-negative length. In order to see the equality relation, note the following: the terms of the first summation cancel each other out. Furthermore, the second summation describes an integral over a closed path in $T^{i}$ which, due to path independence, equals zero.

Weak monotonicity of $f$ and path independence of $E_{-i}\left[\beta^{i}\left(f\left(r^{i}, t^{-i}\right) \mid t^{-i}\right)\right]$ do not imply one another. That weak monotonicity does not imply path independence follows directly from Example 2 and Theorem 4. It can also be derived directly from Example 2. If we consider for example path $A$ consisting of the line segment between $x$ and $y$ and path $\tilde{A}$ consists of the line segment between $x$ and $z$ and the line segment between $z$ and $y$, we find that

$$
\int_{x, A}^{y} \beta(f(r))=-\frac{3}{2} \text { and } \int_{x, \tilde{A}}^{y} \beta(f(r))=3
$$

So the integral of $\beta(f(r))$ from $x$ to $y$ is not independent of the path of integration. That weak monotonicity of $f$ does not imply path independence of $E_{-i}\left[\beta^{i}\left(f\left(r^{i}, t^{-i}\right) \mid t^{-i}\right)\right]$ depends crucially upon the assumption of multi-dimensional type spaces. If we would consider one-dimensional type spaces instead, then weak monotonicity would indeed imply path independence. This can be seen for example in the single-item auction application presented in Section 4 .

That path independence does not imply weak monotonicity is illustrated by the following example.

Example 3 Let us consider the allocation of a single, indivisible object. For simplicity we assume that there exists only a single agent to possibly allocate to. He has a type $t \in T=[0,1]$ which reflects the value of the object for him. Given a report $r$ of the agent, the allocation rule $f: T \mapsto[0,1]$ assigns to him a probability for getting the object. The agent's valuation 
for the resulting allocation is $v(f(r) \mid t)=f(r) t$. Specifically, we set $f(r)=-(2 r-1)^{2}+1$ (see Figure 5). Clearly, $f$ is path independent but not weakly monotone.

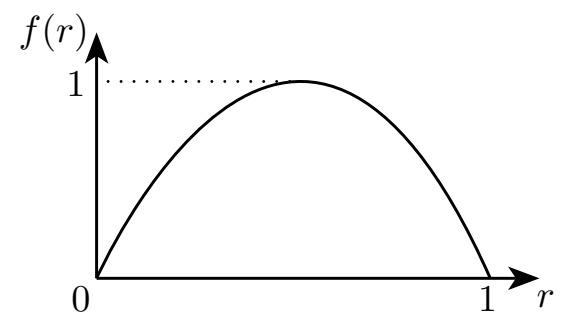

Figure 5: The allocation function in Example 3.

If $f$ is Bayes-Nash incentive compatible, the corresponding payments can be constructed by using shortest path lengths (as described in the proof of Theorem 1). For each $i \in N$, let us pick some $a^{i}$ as the source node in $T_{f}^{i}$. Thus, if agent $i$ reports $t^{i}$, he has to make a payment

$$
P_{i}\left(t^{i}\right)=\inf \sum_{j=1}^{m} l^{i}\left(r_{j}^{i}, r_{j+1}^{i}\right),
$$

where the infimum is taken over all finite paths from $t^{i}$ to $a^{i}$. Take any finite path $A=$ $\left\{r_{1}^{i}=t^{i}, \ldots, r_{m+1}^{i}=a^{i}\right\}$ in $T_{f}^{i}$. Let $L_{j}$ denote the line segment between $r_{j}^{i}$ and $r_{j+1}^{i}$, whereas $L_{t}$ denotes the line segment between the source and $t^{i}$. Following the repeated substitution approach presented in the second part of the proof of Theorem 4, we can construct paths that are shorter (or as long) by letting them visit the same nodes as $A$ and also additional nodes along the line segments inbetween. In the limit, as the number of nodes goes to infinity, the distance between neighboring nodes goes to zero and the length of the paths goes to

$$
\begin{aligned}
\sum_{j=1}^{m}( & E_{-i}\left[v^{i}\left(f\left(r_{j}^{i}, t^{-i}\right) \mid r_{j}^{i}, t^{-i}\right)-v^{i}\left(f^{i}\left(r_{j+1}^{i}, t^{-i}\right) \mid r_{j+1}^{i}, t^{-i}\right)\right] \\
& \left.+\int_{r_{j}^{i}, L j}^{r_{j+1}^{i}} E_{-i}\left[\beta^{i}\left(f\left(r^{i}, t^{-i}\right) \mid t^{-i}\right)\right]\right) .
\end{aligned}
$$


Using path independence (18) we have that ${ }^{12}$

$$
\sum_{j=1}^{m} \int_{r_{j}^{i}, L j}^{r_{j+1}^{i}} E_{-i}\left[\beta^{i}\left(f\left(r^{i}, t^{-i}\right) \mid t^{-i}\right)\right]=\int_{t^{i}, L_{t}}^{a^{i}} E_{-i}\left[\beta^{i}\left(f\left(r^{i}, t^{-i}\right) \mid t^{-i}\right)\right] .
$$

Applying the above to (17) yields

$$
\begin{aligned}
P_{i}\left(t^{i}\right)= & E_{-i}\left[v^{i}\left(f\left(t^{i}, t^{-i}\right) \mid t^{i}, t^{-i}\right)-v^{i}\left(f^{i}\left(a^{i}, t^{-i}\right) \mid a^{i}, t^{-i}\right)\right] \\
& -\int_{a^{i}, L_{t}}^{t^{i}} E_{-i}\left[\beta^{i}\left(f\left(r^{i}, t^{-i}\right) \mid t^{-i}\right)\right],
\end{aligned}
$$

implying that the expected utility (see (1) for definition) for truthfully reporting $t^{i}$ is ${ }^{13}$

$$
U^{i}\left(t^{i} \mid t^{i}\right)=U^{i}\left(a^{i} \mid a^{i}\right)+\int_{a^{i}, L_{t}}^{t^{i}} E_{-i}\left[\beta^{i}\left(f\left(r^{i}, t^{-i}\right) \mid t^{-i}\right)\right]
$$

\subsection{Application to the Social Choice Model of Jehiel \& Moldovanu [4]}

As a special case let us consider the social choice model introduced by Jehiel \& Moldovanu [4] which allows for allocative externalities and interdependent valuations. ${ }^{14}$ In this setting there exists a set of social alternatives $M=\{1, \ldots, m\}$. Given reports from all agents, the allocation rule $f: T \mapsto[0,1]^{m}$ assigns to each social alternative a probability to be chosen. So the outcome set $\Gamma$ is the set of all possible probability profiles. Alternative $k$ 's probability to be chosen, given a report profile $r \in T$, is denoted $f_{k}(r)$. The allocation rule must satisfy the probability conditions $\sum_{k=1}^{m} f_{k}(t)=1$ and $0 \leq f_{k}(t) \leq 1, \forall k \in M, \forall t \in T$.

For every agent $i$, the type space $T^{i} \subseteq \mathbb{R}^{m \times n}$ is assumed to be convex and bounded. Element $t_{k j}^{i}$ of agent $i$ 's type $t^{i} \in T^{i}$ affects agent $j$ 's valuation for social alternative $k \in M$. Agent $i$ 's valuation for some alternative $k \in M$, given a type profile $t \in T$, is $v^{i}(k \mid t)=\sum_{j=1}^{n} a_{k i}^{j} t_{k i}^{j}$, where the scalars $a_{k i}^{j}$ are common knowledge and $a_{k i}^{i} \geq 0, \forall i \in N, \forall k \in M$. Let agent $i$ have true type $t^{i}$ and report $r^{i}$ while the others have true types $t^{-i}$ and reports $r^{-i}$. His valuation

\footnotetext{
${ }^{12}$ The line segment $L_{t}$ for the path of integration is picked for convenience. Due to path independence, it can be replaced with any other path connecting the source and $t^{i}$.

${ }^{13}$ Again, in order to derive (20) one can use that by construction $P_{i}\left(a^{i}\right)=0$ and thus add this term to the right-hand side of (19).

${ }^{14}$ Jehiel, Moldovanu \& Stacchetti [5] present a special case of this model without interdependent valuations. They consider single-item auctions with externalities where agent $i$ has a type $t^{i} \in T^{i} \subseteq \mathbb{R}^{n}$. Type element $t_{i}^{i}$ reflects his valuation for the object, whereas $t_{j}^{i}$ reflects the (commonly negative) value of the externalities agent $i$ incurs if agent $j$ gets the object.
} 
for the resulting outcome is

$$
v^{i}\left(f\left(r^{i}, r^{-i}\right) \mid t^{i}, t^{-i}\right)=\sum_{k=1}^{m}\left(f_{k}\left(r^{i}, r^{-i}\right) \sum_{j=1}^{n} a_{k i}^{j} t_{k i}^{j}\right) .
$$

Assuming that he reports $r^{i}$ and believes that the others report truthfully, his expected conditional probability for social alternative $k \in M$ to be chosen is

$$
q_{k}^{i}\left(r^{i}\right)=\int_{T^{-i}} f_{k}\left(r^{i}, t^{-i}\right) \pi^{-i}\left(t^{-i}\right) d t^{-i} .
$$

Define $Q^{i}\left(r^{i}\right): T^{i} \mapsto \mathbb{R}^{m \times n}$ as the vector field, where, for each $k \in M$, the $k i$ th element is given by $a_{k i}^{i} q_{k}^{i}\left(r^{i}\right)$ and the $k j$ th element is zero $\forall j \neq i$. Agent $i$ 's expected valuation, given report $r^{i}$ and true type $t^{i}$, is

$$
\begin{aligned}
E_{-i}\left[v^{i}\left(f\left(r^{i}, t^{-i}\right) \mid t^{i}, t^{-i}\right)\right] & =\int_{T-i}\left(\sum_{k=1}^{m}\left(f_{k}\left(r^{i}, t^{-i}\right) \sum_{j=1}^{n} a_{k i}^{j} t_{k i}^{j}\right)\right) \pi^{-i}\left(t^{-i}\right) d t^{-i} \\
& =Q^{i}\left(r^{i}\right) t^{i}+\sum_{k=1}^{m} \int_{T-i}\left(f_{k}\left(r^{i}, t^{-i}\right) \sum_{\substack{j \in N \\
j \neq i}} a_{k i}^{j} t_{k i}^{j}\right) \pi^{-i}\left(t^{-i}\right) d t^{-i} .
\end{aligned}
$$

Using (21), the weak monotonicity condition becomes

$$
\left(r^{i}-\tilde{r}^{i}\right)\left(Q^{i}\left(r^{i}\right)-Q^{i}\left(\tilde{r}^{i}\right)\right) \geq 0 \quad \forall i \in N, \forall r^{i}, \tilde{r}^{i} \in T^{i}
$$

Jehiel \& Moldovanu [4] show that a mechanism $(f, P)$ is Bayes-Nash incentive compatible if and only if (22) holds, $Q^{i}$ is path independent $\forall i \in N$ and

$$
U^{i}\left(r^{i} \mid r^{i}\right)=U^{i}\left(\tilde{r}^{i} \mid \tilde{r}^{i}\right)+\int_{\tilde{r}^{i}, S}^{r^{i}} Q^{i}(s) \quad \forall i \in N, \forall r^{i}, \tilde{r}^{i} \in T^{i},
$$

where $S$ denotes a path in $T^{i}$ connecting $\tilde{r}^{i}$ and $r^{i}$. Due to path independence, it does not matter which path of integration is chosen.

Note that in this social choice model an agent's valuation function is linear in his own type. Thus, we can directly apply the results derived earlier in this section. From Theorem 4 it follows that $f$ is Bayes-Nash incentive compatible if and only if the weak monotonicity condition in (22) holds and $Q^{i}$ is path independent $\forall i \in N$. The corresponding payments are given by (19). Thus, if we let $a^{i}$ denote the source node in $T_{f}^{i}$,

$$
P_{i}\left(r^{i}\right)=E_{-i}\left[v^{i}\left(f\left(r^{i}, t^{-i}\right) \mid r^{i}, t^{-i}\right)-v^{i}\left(f^{i}\left(a^{i}, t^{-i}\right) \mid a^{i}, t^{-i}\right)\right]-\int_{a^{i}, S}^{r^{i}} Q^{i}(s),
$$


where $S$ denotes a path in $T^{i}$ connecting $a^{i}$ and $r^{i}$. Due to path independence, it does not matter which path of integration is chosen. This implies (see also (20)) that the expected utility for truthfully reporting $r^{i}$ is

$$
U^{i}\left(r^{i} \mid r^{i}\right)=U^{i}\left(a^{i} \mid a^{i}\right)+\int_{a^{i}, S}^{r^{i}} Q^{i}(s)
$$

thus (23) holds.

In this application, as in the single-item auction application presented in Section 4, the interdependencies with other agents' types enter an agent's valuation function only additively via the $\alpha$-term in (13). However, note that the class of valuation functions presented in (13) also allows for interesting settings where the interdependencies with other agents' types enter multiplicatively via the $\beta$-term. Consider for example the following simple communication network setting:

Example 4 There exist three agents, $N=\{1,2,3\}$, each owning a link in the communication network presented in Figure 6, that is, agent $i$ owns the link $l_{i}$. Furthermore, there exists a social planner who wants to send data from the point of origin to the destination point. In order to do so, he can rent different combinations of links. The outcome set $\Gamma$ contains the possible link combinations he can choose from, $\Gamma=\left\{\emptyset,\left\{l_{3}\right\},\left\{l_{1}, l_{2}\right\},\left\{l_{1}, l_{2}, l_{3}\right\}\right\}$. The planner assigns a value $\delta$ to the successful data transfer. Data can only be sent once. If the planner chooses the link combination $\left\{l_{1}, l_{2}, l_{3}\right\}$, we assume that the following simple rooting policy is employed: with probability $\rho$ the upper connection $\left\{l_{1}, l_{2}\right\}$ is used, and with probability $1-\rho$ the lower connection is used.

Each agent has a type $t^{i} \in[0,1]$ reflecting the probability that his link actually works if the planner tries to send data through it (e.g. it might be busy putting through other data). Furthermore, agent $i$ incurs fixed, publicly known costs $c^{i}$ for putting data through his link. His valuation for $\gamma \in \Gamma$ is $v^{i}\left(\gamma \mid t^{i}\right)=-c^{i} A^{i}(\gamma) t^{i}$ where $A^{i}(\gamma)$ denotes the probability that the data reaches the link $l_{i}$ if outcome $\gamma$ is chosen. The allocation rule of the planner is to pick a $\gamma \in \Gamma$ such that $[\delta T(\gamma)-C(\gamma)]$ is maximized. $T(\gamma)$ denotes the expected throughput, that is, the probability that the data reaches the destination given the link combination $\gamma . C(\gamma)$ denotes the expected throughput costs, that is, $\sum_{i} c^{i} A^{i}(\gamma) t^{i}$. The valuations of the agents for the different outcomes are summarized in Table 1. Note that agent 1's type enters agent 2's valuation of the outcomes $\left\{l_{1}, l_{2}\right\}$ and $\left\{l_{1}, l_{2}, l_{3}\right\}$ in the aforementioned multiplicative fashion. Employing 
different routing policies and data sending strategies, one can construct also examples where each agent exhibits such interdependent valuations.

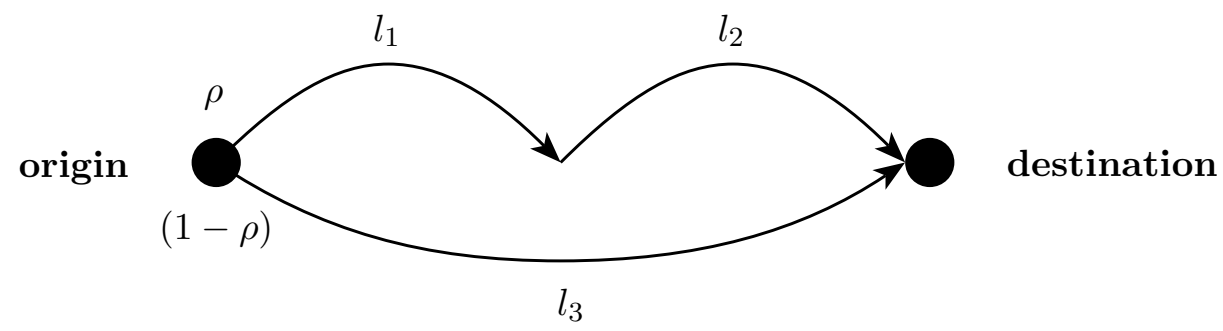

Figure 6: The communication network considered in Example 4.

\begin{tabular}{|l|c|c|c|}
\hline$\gamma$ & $v^{1}\left(\gamma \mid t^{1}\right)$ & $v^{2}\left(\gamma \mid t^{2}\right)$ & $v^{3}\left(\gamma \mid t^{3}\right)$ \\
\hline \hline$\emptyset$ & 0 & 0 & 0 \\
\hline$\left\{l_{3}\right\}$ & 0 & 0 & $-c^{3} t^{3}$ \\
\hline$\left\{l_{1}, l_{2}\right\}$ & $-c^{1} t^{1}$ & $-c^{2} t^{1} t^{2}$ & 0 \\
\hline$\left.\left\{l_{1}, l_{2}, l_{3}\right\}\right\}$ & $-c^{1} \rho t^{1}$ & $-c^{2} \rho t^{1} t^{2}$ & $-c^{3}(1-\rho) t^{3}$ \\
\hline
\end{tabular}

Table 1: Agents' valuations in Example 4.

\section{Acknowledgements}

The authors are grateful to the participants of the Second World Congress of the Game Theory (2004) and the First Spain Italy Netherlands Meeting on Game Theory (2005) for helpful discussions. We especially thank Philip J. Reny and Rakesh V. Vohra for their useful comments.

\section{References}

[1] Ravindra K. Ahuja, Thomas L. Magnanti, and James B. Orlin. Network Flows - Theory, Algorithms and Applications. Prentice-Hall, New Jersey, 1993. 
[2] Sushil Bikhchandani, Shurojit Chatterji, and Arunava Sen. Incentive compatibility in multi-unit auctions. mimeo, June 2003.

[3] Hongwei Gui, Rudolf Müller, and Rakesh V. Vohra. Dominant strategy mechanisms with multidimensional types. METEOR Research Memorandum, available at http://edata.ub.unimaas.nl/www-edocs/, October 2004.

[4] Philippe Jehiel and Benny Moldovanu. Efficient design with interdependent valuations. Econometrica, 69(5):1237-1259, September 2001.

[5] Philippe Jehiel, Benny Moldovanu, and Ennio Stacchetti. Multidimensional mechanism design for auctions with externalities. Jounal of Economic Theory, 85:258-293, 1999.

[6] Paul Klemperer. Auction theory: A guide to the literature. Journal of Economic Surveys, 13(3):227-286, 1999.

[7] Vijay Krishna and Eliot Maenner. Convex potentials with an application to mechanism design. Econometrica, 69(4):1113-1119, July 2001.

[8] Ron Lavi, Ahuva Mu'alem, and Noam Nisan. Towards a characterization of truthful combinatorial auctions. In Proceedings of the 44th Annual IEEE Symposium on Foundations of Computer Science (FOCS'03), 2003.

[9] Wilhelm Maak. Differential- und Integralrechnung. Vandenhoeck \& Ruprecht, Göttingen, 4th edition, 1969.

[10] Alexey Malakhov and Rakesh V. Vohra. Single and multi-dimensional optimal auctions - a network approach. mimeo, September 2004.

[11] Roger B. Myerson. Optimal auction design. Mathematics of Operations Research, 6(1):58-73, February 1981.

[12] Jean-Charles Rochet. A necessary and sufficient condition for rationalizability in a quasilinear context. Journal of Mathematical Economics, 16:191-200, 1987.

[13] R. Tyrrell Rockafellar. Characterization of the subdifferentials of convex functions. $P a-$ cific Journal of Mathematics, 17(3):487-510, 1966. 
[14] R. Tyrrell Rockafellar. Convex Analysis. Princeton University Press, Princeton, New Jersey, 1970.

[15] Michael Saks and Lan Yu. Weak monotonicity suffices for truthfulness on convex domains. In Proceedings of the 6th ACM Conference on Electronic Commerce (EC'05), pages 286-293, 2005.

[16] Paul Shostak. Deciding linear inequalities by computing loop residues. Journal of the ACM, 28(4):769-779, October 1981. 